\title{
$\mathrm{P}$ is for Phenomenology
}

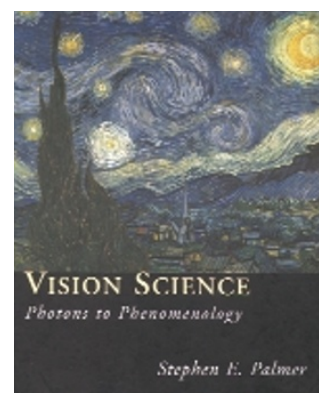

\section{Vision Science: Photons to Phenomenology}

by Stephen E. Palmer

The MIT Press, Cambridge, Massachusetts, 1999. $\$ 70.00$ Cloth, pp 810

ISBN 0-262-16183-4

\section{Reviewed by Karl R. Gegenfurtner}

One of the goals of neuroscience is to integrate different disciplines that used to be relatively unrelated, but all study the nervous system. Vision science has been at the forefront of the neuroscience movement, and some of the credit for this should go to the late David Marr. His 1982 book, Vision, smoothly integrated psychophysics, physiology and computation into a comprehensive theory of how vision works. Stephen Palmer's Vision Science fits right into Marr's framework. It is a comprehensive and excellent textbook on visual perception with the specific purpose of reflecting the interdisciplinary nature of visual science.

The older textbooks on visual perception were conceived strictly for an audience of psychology students, and consisted mainly of descriptions of fascinating but often unrelated and unexplained phenomena. More recent textbooks had the look and feel of introductions to sensory physiology. What was missing from all these books was the 'glue' linking phenomena, physiology and computation. Stephen Palmer has put forward his attempt at an integrated overview of the field, and he probably has done his job as well as any person could possibly do it. The volume of the book is also impressive-it has about twice as many pages as previous textbooks devoted to vision.

There are two striking aspects to this book. First, despite its title, there is a clear dominance of phenomenology, and hardly anything about photons. Second, the best chapters are the ones on higher-level visual processes, such as perceptual organization, object recognition, visual attention and visual memory. Although the book is meant to be an undergraduate textbook for the whole field of vision, it

Karl Gegenfurtner is at MPI für biologische

Kybernetik, Spemannstrasse 38, 72076

Tübingen, Germany.

email:karl@kyb.tuebingen.mpg.de would be just as appropriate as a graduate text for a course on visual cognition. Presumably this reflects the influence of the author's mentor, the late Irving Rock, famous for his work on perceptual illusions; indeed, Palmer himself is best known for his studies of perceptual organization.

The organization of Vision Science is somewhat unusual. Rather than dividing the subjects into different chapters dealing with distinct visual attributes such as color, form, motion and depth, the book is split into two major parts-spatial vision and visual dynamics. There is one exception; the chapter on color vision precedes all others, with the justification that color vision is exemplary for illustrating the interdisciplinary nature of vision science. I fully agree with this, and, because color vision is my main area of expertise, I was looking forward to an integrative review of what physics, physiology, biology and psychology had come up with during the last 100 years. What I found, however, was disappointing, in that it did not offer any justification for the major claim that its integrated and interdisciplinary nature makes color vision a 'microcosm' of vision science.

To start with, Palmer introduces a purely phenomenological color space, based on lightness, hue and saturation. Yet nearly all contemporary research on color vision uses color spaces based on the physiological signals either in the cone photoreceptors or in the subsequent color-opponent retinal ganglion cells. In both cases, there is a clear physiological substrate, making it easy to relate psychophysical findings to physiology. As a result of this inappropriate choice, students will be ill-prepared to read the current literature on color vision.

There are other problems (most of which occur in other textbooks as well) with the color vision chapter. The 'unique hues' postulated by Hering and by Hurvich and Jameson are mistaken for the wellestablished color-opponent channels in the retina. Also, the textbook myth of double- opponent cells in the visual cortex of primates is perpetuated here. Even though such cells offer an elegant solution to most problems of color contrast and constancy, since 1978 nobody has been able to verify their existence in primate visual cortex.

Luckily for the student (and teacher), most of the other chapters are of much higher quality. The two introductory chapters on 'Foundations' were particularly pleasing. They offer an overview of current thinking and a quick history of the field over the last 50-100 years. The chapter on depth perception contains some of the latest psychophysical findings and computational arguments on how to combine information from different depth cues ('sensor fusion'). The chapter on perceptual organization introduces computational arguments to a field that is typically regarded as vague and full of hand-waving arguments. Similarly, the chapter on object recognition is focused on the current debate between imagebased approaches and recognition by three-dimensional components. There is a chapter on illusions and constancies that is amazingly complete. The materials on visual attention and eye movements, visual memory and imagery and awareness are also welcome additions that are rarely found in perception textbooks.

Of course, most of these chapters cover perceptual phenomena for which there are no immediate links to physiology, and little relationship to computation. The best chapters of the book are the ones in which phenomenology dominates. Chapters on lower-level processes are of lower quality. This is reflected not only in the material that is presented, but also in what is missing. In my view, the two biggest advances in visual science over the last decade were the discovery of the molecular genetic basis of color vision by Jeremy Nathans and colleagues, and the microstimulation experiments of Bill Newsome and colleagues. These experiments establish a direct link between genetics and behavior, and between responses of single neurons and behavior, respectively. If the motivation behind this textbook was to show an integrated picture of visual science, then these two landmark discoveries should have been included.

Even so, it is difficult to argue that there is material missing in a book that is already 800 pages thick, and the above criticisms hold for most other undergraduate textbooks as well. As it stands, Vision Science is the best and most complete current textbook on visual perception. I expect it to become the classroom standard. 